\title{
New surgical modification of fascial closure following endovascular aortic pathology repair
}

\author{
Miroslaw Dziekiewicz¹, Rafał Maciag², Marek Maruszynski \\ 1Department of Vascular and Endovascular Surgery, Military Institute of Medicine, Warsaw, Poland \\ 2Second Department of Clinical Radiology, Warsaw Medical University, Poland
}

Videosurgery Miniinv (e-pub, ahead of print) DOI: 10.5114/wiitm.2011.35795

\begin{abstract}
Introduction: There are clear benefits of percutaneous versus open femoral access for endovascular aortic pathology repair. All closing devices $(C D)$ commercially available are expensive. Surgical closure of the femoral artery risks potential prolonged wound healing and as a consequence longer hospital stay. Fascial closure is a technique that remains an interesting option.

Aim: To evaluate the efficacy of the surgical modification of hemostasis control after endovascular repair of aortic pathology.

Material and methods: One hundred sixteen common femoral arteries in a group of 58 patients underwent a minimally invasive procedure. Patients suffering from abdominal (AAA), thoracic aorta aneurysms (TAA), acute thoracic aorta type $B$ dissections $(A A D)$ and traumatic aortic injury (TAI) were treated.

Results: A 1-year period of experience in fascial closure of 116 common femoral arteries (CFA) was presented in the group of 58 patients undergoing endovascular interventions. Five intraoperative complications were observed and one late. Three primary failures were due to hemorrhage in three arteries, one required open repair and two additional compression after the procedure. Two cases of limb ischemia required surgical correction of artery closure. One limb ischemia was detected 4 weeks later, and was treated conservatively. At 1 year, 92 fascial closures (80\%) were in the follow-up and 24 (20\%) were lost to follow-up.

Conclusions: This new modification of fascial closure is a safe and cheap method of arterial closure following endovascular repair of selected aortic pathologies. The usage of two suture lines makes this procedure easy and quick. Fascial closure technique is comparable to other techniques in terms of success and complication rates.
\end{abstract}

Key words: endovascular procedure, fascial closure, closing device.

\section{Introduction}

There is no question that percutaneous access for aortic pathology treatment offers a clinical benefit. Endovascular procedures give the possibility of minimally invasive methods for treatment of life-threatening conditions such as thoracic aorta aneurysms (TAA), abdominal aorta aneurysms (AAA), acute tho- racic aorta type $B$ dissections ( $A A D$ type $B$ ) and traumatic aortic injury (TAI). Minimization of surgical trauma, blood loss, post-operative pain and complications resulting from surgical access to the groin, e.g. an infection or seroma formation [1-3], are clear benefits of this procedure. To complete a fully percutaneous intervention different CDs are proposed. In addition to complications typical for this method, 
economic factors still remain to be overcome. The fascial closure technique presented by Larzon et al. [4] seems to be a quite good solution. Early complications associated with this method may be hemorrhage or thrombosis. Arterial stenosis or occlusion and pseudoaneurysm formation may be found mainly as late complications. In this study the authors present the results of percutaneous endovascular aortic pathology repair with their own modification of fascial closure of femoral artery puncture.

\section{Aim}

The aim was to assess the early and late complications.

\section{Material and methods}

The general steps of this technique were precisely described by Larzon et al. [4] and Löhn et al. [5]. First, our modification was an accurate assessment of femoral artery position based on computed tomogra-

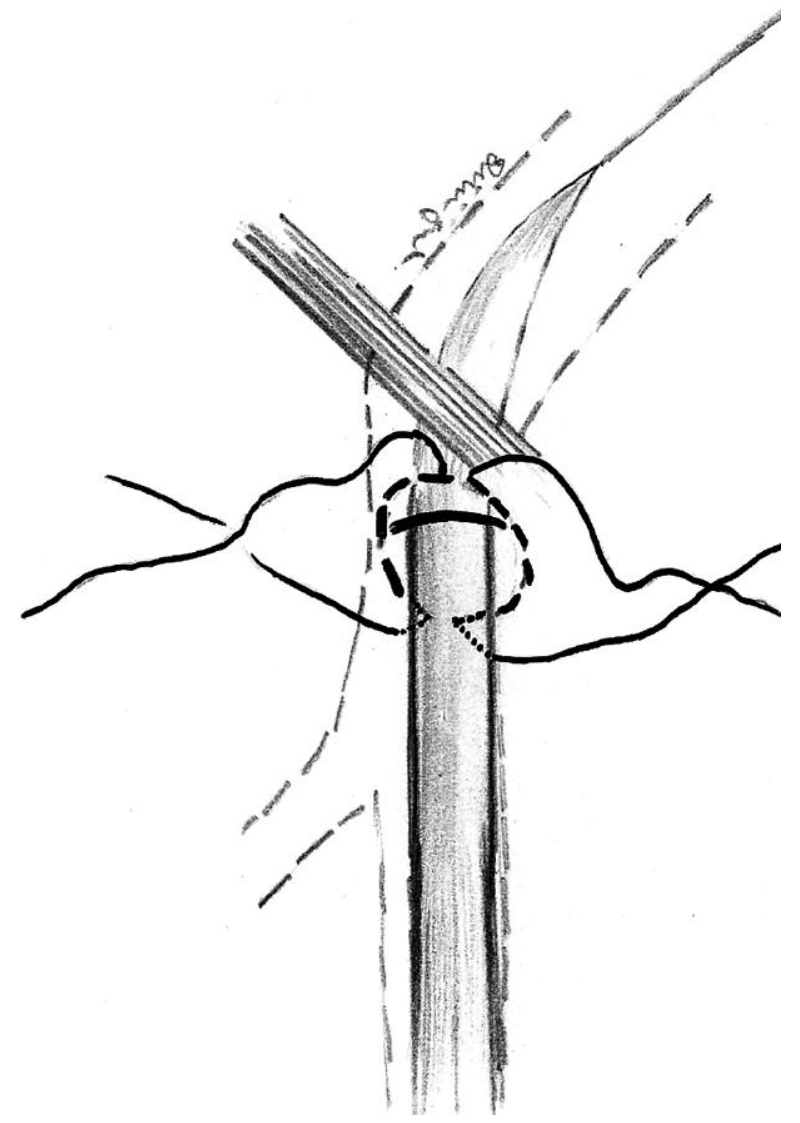

Figure 1. The scheme of fascial closure technique phy angiography (CTA) according to accurate evaluation of the anatomical topography. Next, continuing the procedure femoral artery puncture was done, and the guide wire and introducer were introduced in situ. A longitudinal skin incision of 3-4 cm was made and dissected tissues - the cribriform fascia - were visualized. A double U-shaped suture line (Prolene 2-0) was placed from both sides of the lumen of the introducer (Figure 1). In the end, the sutures were tightened, tamponading the bleeding, as the introducer was gradually removed, leaving the guidewire in the lumen of the artery. If hemostasis was obtained, the guidewire was removed, the sutures tightened to the end. The skin sutures completed the procedure. If bleeding occurred, the introducer was repositioned to stop bleeding and next a superficial Z-shape suture (Prolene 3-0) was added. If it was necessary, open dissection of the femoral artery was performed with arterial closure. Fascial closure technique did not prolong postoperative patient mobilization and the hospital stay. The patients obtained postoperative antiplatelet therapy and deep vein thrombosis prophylaxis according to the relevant recommendations.

Endovascular treatment was performed in 58 patients who were recruited to this trial; 116 femoral arteries were closed between November 2011 and September 2012. A minimum of two consultants analyzed patients and their pre-operative CTA, taking into consideration intra-operative findings. All inclusion and exclusion criteria were strictly adhered to in this trial. It means that morbid obesity represented an obvious contraindication. Also a high common femoral artery (CFA) bifurcation or puncture of the external iliac artery made fascial closure impossible to do lege artis. Surgical interventions in this region in anamnesis could also be an important factor that should be taken into consideration prior to surgery. The diameter of CFA $<6 \mathrm{~mm}$ created an independent risk factor of safe artery closure. An accommodation of sheaths used during all of the operations could be difficult and resulted in the necessity of surgical reconstruction of the artery. In every case the surgeon was ready to use one of all alternative methods to close an artery puncture. On the ipsilateral artery sheath the inner diameter was up to $24 \mathrm{Fr}$ and on the contralateral side 12-16 Fr were used. The primary evaluation of the procedure was technical success resulting in hemostasis. The group of secondary outcomes consisted of hematoma followed by evacuation, thrombosis requiring further surgery, wound 
infection, seroma, pseudoaneurysm formation and in the common femoral artery stenosis or occlusion. None of them were detected in our group of patients. Clinical evaluation and duplex scan or CTA if needed were performed at 1 month, 3 months, 6 months and once a year. Duplex and CTA were evaluated by an experienced radiologist and vascular surgeon. The follow-up protocol was the same for all patients who underwent endovascular intervention in our department and focused on the outcome of fascial closure.

\section{Results}

Between November 2011 and October 2012, during the study period Thoracic Endovascular Aortic Repair (TEVAR), Endovascular Aneurysm Repair (EVAR), endovascular treatment of ADD type $B$ and TAI, were performed in 58 patients, in whom 116 fascial closure procedures of CFA were done. Mean age was 52 years (range 21-83 years), 5 female (9\%). Only results for included patients are presented. All patients underwent total percutaneous endograft placement using fascial closure technique to control hemostasis on both sides - i.e. right end of left CFA. A successful primary outcome was observed in 111 arteries (96\%). Primary failure was noted in 5 (4.3\%) patients. Every time it was only one side, which required additional procedures. Intra-operatively 3 (2.6\%) hemorrhages were revealed. Two of them required only external hemostatic compression. One femoral artery was sutured. Two (1.7\%) patients presented limb ischemia symptoms in a few hours after completing the procedure. They necessitated open cut down and repair. One symptomatic right CFA stenosis $(0.8 \%)$ occurred 1 month after the procedure. The patient developed claudication (200 m). She was treated conservatively. Three (2.6\%) patients had access side pseudoaneurysm and all of them resolved within the period of observation. None required secondary intervention. There were no superficial wound complications, venous complications or femoral nerve neuralgia. No patients required a secondary intervention related to fascial closure technique (excluding one woman who did not agree for PTA of stenosed CFA). All patients underwent Duplex or CTA in accordance with the above-mentioned time points. Four (3.4\%) patients required PTA of superficial femoral artery occlusion. It was also found on pre-operative CTA. It means that there was no connection with the performed proce- dure. No further complications were found in the observation period.

\section{Discussion}

The results presented in this article confirm a technical success rate of $96 \%$ of the modification of fascial closure technique. All surgical indications for the endovascular procedures were focused on totally percutaneous minimally invasive procedure. Looking at the final results we can suggest this technique as safe and effective. Our experience has shown that an accurate selection of appropriate patients and intraoperative care permitted us to avoid or minimize the complication rate. As we have seen, what we were worried about at the beginning, e.g. stenosis, occlusion, or pseudoaneurysm formation, was not a significant problem. Our early experience in this field showed that our modification of fascial closure technique remains a safe alternative not only to open cut down but others' experience as well e.g. with closing devices. The benefits of this technique are clearly expressed by a reduction of postoperative pain, seroma formation and on the other hand shortened duration of the procedure. We do not wait for exposure of vessels in the groins, and after completing the procedure the use of the proposed technique is a quick and successful way to control hemostasis. The results obtained in this study are similar to those reported by Larzon et al. [4] and Harrison et al. [6] with the same level of technical success. Eight patients treated by Larzon et al. [4] were taken back to the operating room. As Harrison et al. mentioned, one of the crucial steps during the procedure was to leave a guidewire in the artery lumen until satisfactory hemostasis was provided. In every case the sheath could be replaced and the artery sutured in a classical way [6]. The use of closing devices permitted a success rate of $94 \%$ to be gained and long-term complication rates of $1 \%$ associated with the Proscar [7] and Proglide device [8]. Both cited results proved good technical success with minimal complication rates of a wholly percutaneous approach. However, the application of CD significantly increased the cost of treatment. In terms of cost, fascial closure technique is much more economical. The rate of pseudoaneurysms gained the level of $1 \%$ following fascial closure in the experience of Larzon et al. [4] and even $2 \%$ following the closing device in the work of Starnes et al. [7]. These results could be discussed because pseudoaneurysms greater than 
$3 \mathrm{~cm}$ were included in the complication rate. In our experience the incidence of pseudoaneurysms associated with the fascial closure technique is similar to the others' results comparing different methods of totally percutaneous interventions. We also have not observed femoral nerve neuralgia, which could be a potential further complication. We conclude that it was possible by careful dissection of tissues with identification of femoral fascia in the surroundings of the femoral artery puncture.

This method also allowed the length of the cutting line to be minimized. From an esthetic point of view it can be a serious factor of intervention, especially in young women. Next, re-interventions through both a percutaneous and open approach could be easier to accomplish in the reduced scarring area. It is not a rare problem to gain adequate access to the femoral artery after several open or percutaneous interventions et al. [6]. We did not observe problems with wound healing, e.g. infections or seroma formation [6]. The results were close to those obtained by Harrison [6]. 8.5\% wound infection rate and $4.8 \%$ rate of lymphocele formation remain acceptable. Femoral nerve and femoral artery complications may occur but most of all at the beginning of the personal surgeon's experience with this technique. For this reason, in our department almost all fascial closure procedures were performed by one surgeon, except four - made by the other specialists in assistance of the first one. Even a small incision between $2.5 \mathrm{~cm}$ and $5 \mathrm{~cm}$ allowed an adequate visualization of the fascia lata in the bottom of the wound and the inguinal ligament in the upper part of the incision. After achieving these conditions, accurate placement of the sutures is quite an easy and safe maneuver.

The CTA of all patients were analyzed to avoid massive calcification of the femoral artery. The artery lumen diameter was compared to sheath size. Severe calcification associated with an artery lumen decrease was a relative contraindication for the fascial closure technique or CD's usage. Those patients were candidates for an endarterectomy and profundoplasty.

The limitations to this study include not a large group of patients who underwent interventions through the femoral artery, massive atherosclerotic changes in the CFA or small diameter of the CFA. This last feature is quite common in women. However, in our group of patients there were $9 \%$ women.

The fascial closure technique is a quite new way to safely close an arterial puncture [4, 9-11] and because of this it should be compared with other techniques of CFA closure including open cut down and percutaneous CDs. Only a randomized trial could compare all methods, including a cost analysis with the evaluation of the technique related to inclusion and exclusion criteria values. Our method of suture making seems to be also effective and appears to be an interesting alternative to those presented by other authors.

\section{References}

1. Minion DJ, Davenport DL. Access techniques for EVAR: percutaneous techniques and working with small arteries. Semin Vasc Surg 2012; 25: 208-16.

2. Torsello GB, Kasprzak B, Klenk E, et al. Endovascular suture versus cutdown for endovascular aneurysm repair: a prospective randomized pilot study. J Vasc Surg 2003; 38: 78-82.

3. Slappy AL, Hakaim AG, Oldenburg WA, et al. Femoral incision morbidity following endovascular aortic aneurysm repair. Vasc Endovascular Surg 2003; 37: 105-9.

4. Larzon T, Geijer H, Gruber G, et al. Fascia suturing of large access sites after endovascular treatment of aortic aneurysms and dissections. J Endovasc Ther 2006; 13: 52-7.

5. Löhn L, Larzon T, Van Den Berg JC. From puncture to closure of the common femoral artery in endovascular aortic repair. J Cardiovasc Surg 2010; 51: 791-8.

6. Harrison GJ, Thavarajan D, Brennan JA, et al. Fascial closure following percutaneous endovascular aneurysm repair. Eur J Vasc Endovasc Surg 2011; 41: 346-9.

7. Starnes BW, Andersen CA, Ronsivalle JA, et al. Totally percutaneous aortic aneurysm repair: experience and prudence. J Vasc Surg 2008; 47: 919-23.

8. Lee WA, Brown MP, Nelson PR, et al. Midterm outcomes of femoral arteries after percutaneous endovascular aortic repair using the Preclose technique. J Vasc Surg 2008; 47: 919-23.

9. Arthurs ZM, Starnes BW, Sohn VY, et al. Ultrasound-guided access improves rate of access-related complications for totally percutaneous aortic aneurysm repair. Ann Vasc Surg 2008; 22: 736-41.

10. Eisenack M, Umscheid T, Tessarek J, et al. Percutaneous endovascular aortic aneurysm repair: a prospective evaluation of safety, efficiency, and risk factors. J Endovasc Ther 2009; 16: 708-13.

11. Dalainas I, Nano G, Casana R, Tealdi D. Mid-term results after endovascular repair of abdominal aortic aneurysms: a four-year experience. Eur J Vasc Endovasc Surg 2004; 27: 319-23.

Received: 9.02.2013, accepted: 4.04.2013. 